\title{
Treatment of Macular Degeneration with Sildenafil: Results of a Two-Year Trial
}

\author{
D. Jackson Coleman ${ }^{a}$ Winston Lee ${ }^{a}$ Stanley Chang ${ }^{a}$ Ronald H. Silverman ${ }^{a}$ \\ Harriet O. Lloyd ${ }^{a}$ Suzanne Daly ${ }^{a}$ Stephen H. Tsang ${ }^{a-c}$ \\ aDepartment of Ophthalmology, Columbia University, New York, NY, USA; ${ }^{b}$ Jonas Children's Vision Care and \\ Bernard \& Shirlee Brown Glaucoma Laboratory, Columbia University, New York, NY, USA; 'Department of \\ Pathology and Cell Biology, Institute of Human Nutrition, College of Physicians and Surgeons, Columbia University, \\ New York, NY, USA
}

\section{Keywords}

Macular degeneration · Sildenafil · Viagra ·

Phosphodiesterase $\cdot$ Warburg glycolysis

\begin{abstract}
Objective: To evaluate PDE5/6 inhibition with sildenafil to reduce choroidal ischemia and treat age-related macular degeneration. Methods: Sildenafil was prescribed to treat participants with macular degenerations or macular dystrophies measured by spectral-domain optical coherence tomography, color fundus photography, enhanced depth imaging, and best-corrected visual acuity. Results: No change in calcified drusen was noted. Vitelliform-type soft drusen were not substantially changed. A participant with Best vitelliform macular dystrophy had a significant improvement in vision as well as in photoreceptor and ellipsoid layers. Conclusions: Our research supports sildenafil as a safe treatment for age-related and vitelliform macular degenerations. Thickened Bruch's membrane reduces the beneficial effect of perfusion increase, but all eyes appear to benefit from PDE6. Notably, maintenance or improvement in
\end{abstract}

\section{KARGER}

(c) 2018 S. Karger AG, Basel

E-Mail karger@karger.com

www.karger.com/oph the photoreceptor layer may be the most significant result of sildenafil and is consistent with PDE6 inhibition. Thus, sildenafil treatment of macular degeneration offers significant potential for vision retention and recovery.

(c) 2018 S. Karger AG, Basel

\section{Introduction}

Successful treatment and reversal of age-related macular degeneration (AMD) remains elusive. Though it may remain stable for long periods of time, dry AMD is considered incurable, the only present treatment being the use of vitamins and supplements as described in the AgeRelated Eye Disease Study [1, 2].

Several investigators, including Hayreh [3], Friedman and Oak [4], Flower et al. [5], and Grunwald et al. [6], have proposed that ischemia is a leading cause of AMD. Our team, using wavelet ultrasound technology, has validated the concept that choroidal ischemia is present in nearly all cases of dry macular degeneration [7]. AMD is a multifactorial disease, but unlike other 
Table 1. Clinical and treatment summary of the study cohort

\begin{tabular}{lllllll}
\hline $\begin{array}{l}\text { Patient } \\
\text { No. }\end{array}$ & $\begin{array}{l}\text { Age, } \\
\text { years }\end{array}$ & Sex & Condition & Clinical feature & $\begin{array}{l}\text { Treatment } \\
\text { interval }\end{array}$ & $\begin{array}{l}\text { Sildenafil dose, mg } \\
\text { (morning/evening) }\end{array}$ \\
\hline 1 & 73 & F & dry AMD & foveal drusen & 24 months & $20 / 40$ \\
2 & 82 & M & dry AMD & foveal drusen & 24 months & $20 / 20$ \\
3 & 72 & F & dry AMD & acquired vitelliform & 24 months & $20 / 20$ \\
4 & 59 & M & dry AMD & acquired vitelliform & 24 months & $40 / 40$ \\
5 & 29 & F & Best disease & vitelliform & 24 months & $20 / 20$ \\
\hline
\end{tabular}

AMD, age-related macular degeneration.

causes such as genetic or oxidative stress, ischemia can be treated. Choroidal perfusion and thickness have both been shown to increase in response to systemic sildenafil [8]. While it is intuitively obvious that thickness of the choroid alone does not guarantee better choriocapillaris oxygenation (e.g., the choroid in myopia is often very thin), it is a reasonable step towards ameliorating ischemia in dry AMD.

Systemic treatment using a PDE5 and PDE6 inhibitor (sildenafil) is suggested as a means of increasing choroidal perfusion. Kim et al. [8], using ultrasound, demonstrated that sildenafil can produce increased choroidal perfusion, and Vance et al. [9], using optical coherence tomography (OCT), demonstrated that sildenafil produced increased choroidal thickening. Sildenafil $50 \mathrm{mg}$ has also been shown to increase both blood flow velocity in the ophthalmic artery [10] and perfusion, using Doppler ultrasound imaging $[11,12]$.

In a small off-label series of patients with macular degeneration treated with sildenafil, we noted no loss of vision over 2 years. These encouraging results led us to propose an institutional review board-approved study. Based on the hypothesis of increasing choroidal perfusion as a means of elevating nitric oxide (a messenger molecule) transfer across Bruch's membrane by PDE5 as well as the increase in photoreceptor regeneration caused by a decrease in Warburg glycolysis by PDE6, we treated a series of macular degeneration patients with systemic sildenafil. Our objective in this small pilot, nonrandomized study was to document the effects of sildenafil as well as PDE5 and PDE6 on the photoreceptor layers as well as the accumulation and reduction of lipid material. This small series was designed to develop a proof of concept over a carefully documented 24-month period. Representative findings are presented.

\section{Methods}

Patients with a variety of macular degeneration representing each of the three major phenotypes (dry age-related with drusen, vitelliform, and AMD-like dystrophies) were enrolled in our Health Insurance Portability and Accountability Act-compliant, institutional review board-approved study, which Columbia University agreed upheld the tenets of the Declaration of Helsinki.

Participants with macular degeneration were identified from the investigators' practices at the Edward S. Harkness Eye Institute. After participants had provided informed consent, they were tested and prescribed sildenafil citrate (sildenafil). Doses were determined by body weight; participants weighing $\leq 150$ lbs were prescribed $40 \mathrm{mg}$ b.i.d. ( $20 \mathrm{mg}$ in the morning, $20 \mathrm{mg}$ in the afternoon), and participants weighing $>150 \mathrm{lbs}$ were prescribed up to $80 \mathrm{mg}$ b.i.d. (20-40 $\mathrm{mg}$ in the morning, $20-40 \mathrm{mg}$ in the afternoon). This was based on established, FDA-approved treatment dosages for pulmonary arterial hypertension [12].

Baseline testing included best-corrected visual acuity (BCVA), contrast sensitivity with the Pelli-Robson chart, super-continuum OCT (line/volume), spectral-domain OCT (SD-OCT) with enhanced depth imaging (choroid), OCT angiography, and color fundus photography. These measurements were repeated after initiation of sildenafil, at month 1 , at month 2 , and then bimonthly for the duration of the study. Since it has been shown that patients using nitrates for cardiac conditions could potentially experience substantial hypotension on sildenafil, such patients were excluded from the study. Otherwise, the ocular safety of sildenafil in this dosage range has been well established in studies by Wirostko et al. [13] and by Cordell et al. [14].

\section{Results}

The study cohort consisted of 4 patients who exhibited changes within the clinical spectrum of AMD: soft and hard drusen (patients 1 and 2; mean age 77.5 years) and acquired vitelliform lesions in the fovea (patients 3 and 4; mean age 65.5 years). Best disease was genetically confirmed in patient 5 . The baseline demographic, clinical, 


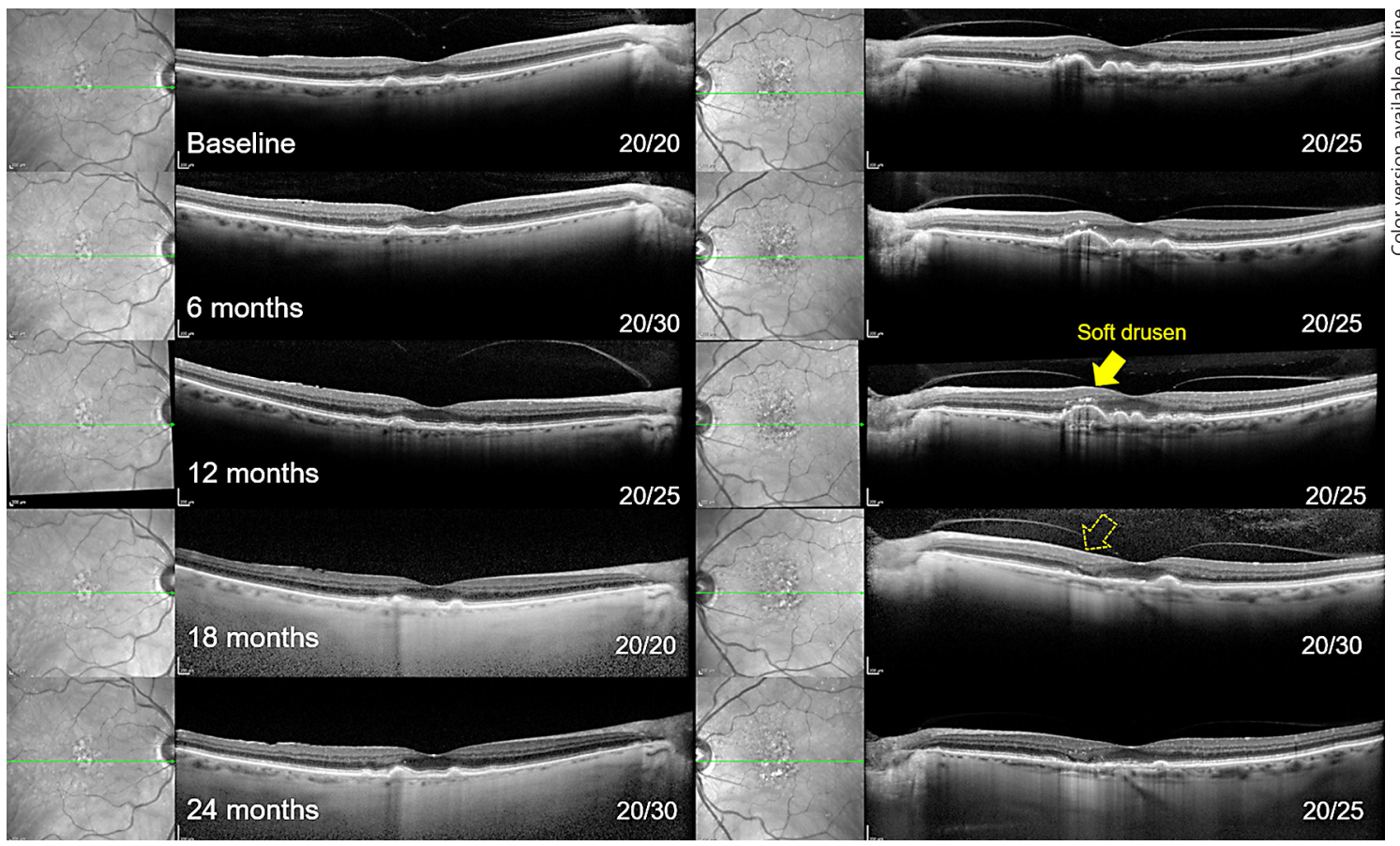

Fig. 1. Serial spectral-domain optical coherence tomography imaging from baseline (pre-sildenafil) to 24 months in 6-month intervals in patient 1 . Drusen are apparent and remain stable along with best-corrected visual acuity in the right eye (left column), while a noted change of a soft druse is observed in the left eye which has resorbed at the 24-month exam (yellow arrow, right column).

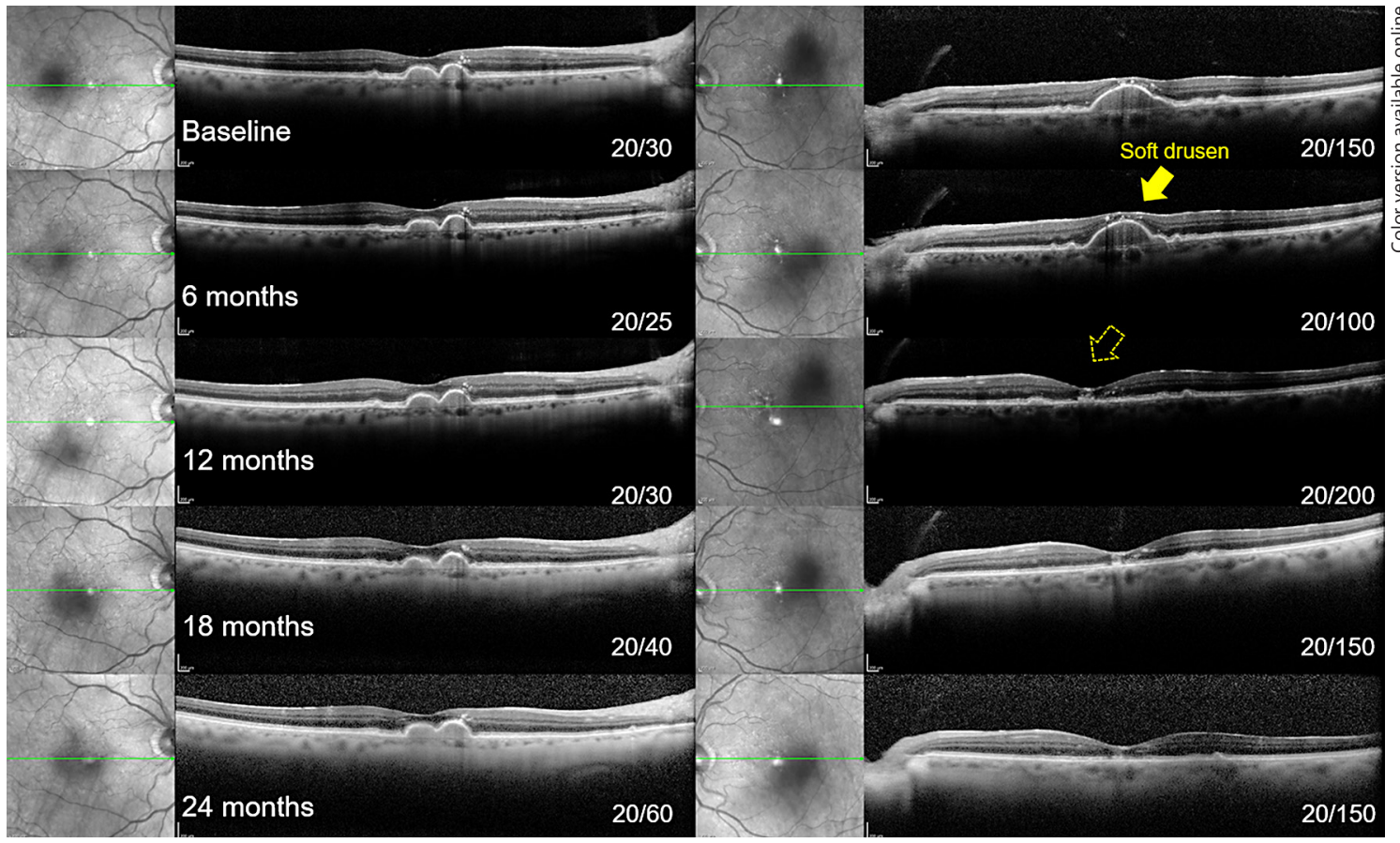

Fig. 2. Serial spectral-domain optical coherence tomography imaging from baseline (pre-sildenafil) to 24 months in 6-month intervals in patient 2. Soft drusen are apparent and remain stable along with best-corrected visual acuity in the right eye (left column), while a noted resorption of a large foveal druse is observed in the left eye (yellow arrow, right column) at the 12-month exam.

Sildenafil Treatment for Macular Degeneration
Ophthalmologica

DOI: $10.1159 / 000486105$ 

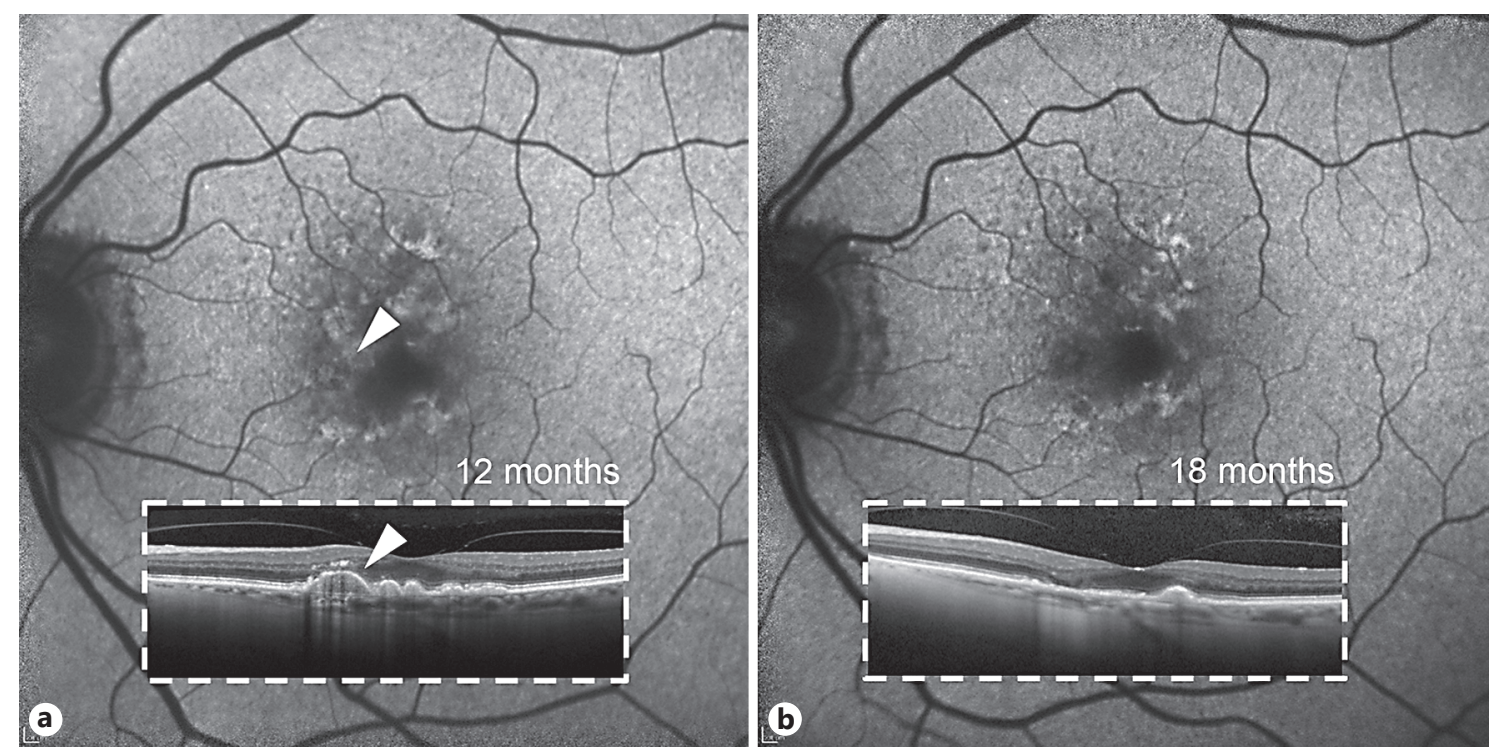

Fig. 3. Autofluorescence correlation of the soft druse change (white arrowhead) in patient 1 at 12 months (a) and at 18 months (b). A residual area of hypoautofluorescence is noted at the area of the soft druse resorption.

and treatment parameters are summarized in Table 1 . A 24-month follow-up period, across four 6-month intervals, is presented for each patient. BCVA at presentation was 20/20 OD and 20/25 OS in patient 1 and 20/30 OD and 20/150 OS in patient 2. At baseline, patient 1 exhibited a pronounced distribution of drusen in the left eye as compared to the right eye, which resulted in reduced visual acuity in the former. Large soft drusen deposits ( 0.5-1.2 mm diameter) were apparent in both eyes of patient 2 , resulting in similarly poor visual acuity, particularly in the left eye, although stationary floaters obscuring vision in proximity to the macula may have been a contributing factor. Hyperreflective deposits above the discernably larger foveal drusen were observed in both patients and resolved at 6 months in patient 2 and at 12 months in patient 1 (Fig. 1, 2, yellow arrows). Resolution of the large drusen in patient 1 resulted in a well-delineated hypoautofluorescence region on autofluorescence imaging (Fig. 3). Visual acuity in both eyes remained relatively stable over this period, with minimal diminishment in the right eye of patient 2 .

Bilateral, subretinal accumulations of vitelliform lipid ranging between 1.2 and $2.5 \mathrm{~mm}$ in diameter were observed on fundoscopy and SD-OCT in the fovea of patient 3 (Fig. 4) and patient 4 (Fig. 5). The lesions exhibited an increased signal on autofluorescence imaging and were moderately asymmetrical in size, larger in the right eye of patient 3 and larger in the left eye of patient 4 . No- tably, an isolated region of the ellipsoid zone band over the vitelliform was observed in both patients, except at the borders (Fig. 3). Visual acuity remained stable at 20/20 to $20 / 25$ in both eyes of patient 4 , whereas moderately decreased visual acuity was measured in the right eye of patient 3, which exhibited a larger accumulation. Acuity remained stable over the 24 -month period. SD-OCT imaging revealed an increase in drusenoid deposition within and proximal to the vitelliform lesion in patient 4; however, the ellipsoid zone layer directly above the vitelliform accumulation remained visible on SD-OCT.

A 29-year-old patient with Best vitelliform macular dystrophy is presented in Figure 6, with large foveal vitelliform lesions bilaterally. At presentation, the lesions were observed to be in the pseudohypopyon stage (III) in the right eye and in the vitelliform stage (II) in the left eye (Fig. 6). BCVA was preserved in the right eye, but reduced in the left eye at 20/150. The vitelliform lipid in the right eye exhibited minor changes, but persisted throughout the 24-month period. The left eye progressed to the vitelliruptive stage (IV) at 6 months and to the atrophic stage (V) at 12 months which, unsurprisingly, coincided with a significant drop in vision (20/400) (Fig. 7). Profoundly significant however was a recovery and persistence of the displaced ellipsoid zone band (photoreceptor) layer over the vitelliform lesion in the left eye (Fig. 7) and the right eye (Fig. 8) along with an improvement of BCVA to 20/100 (from 20/400). 


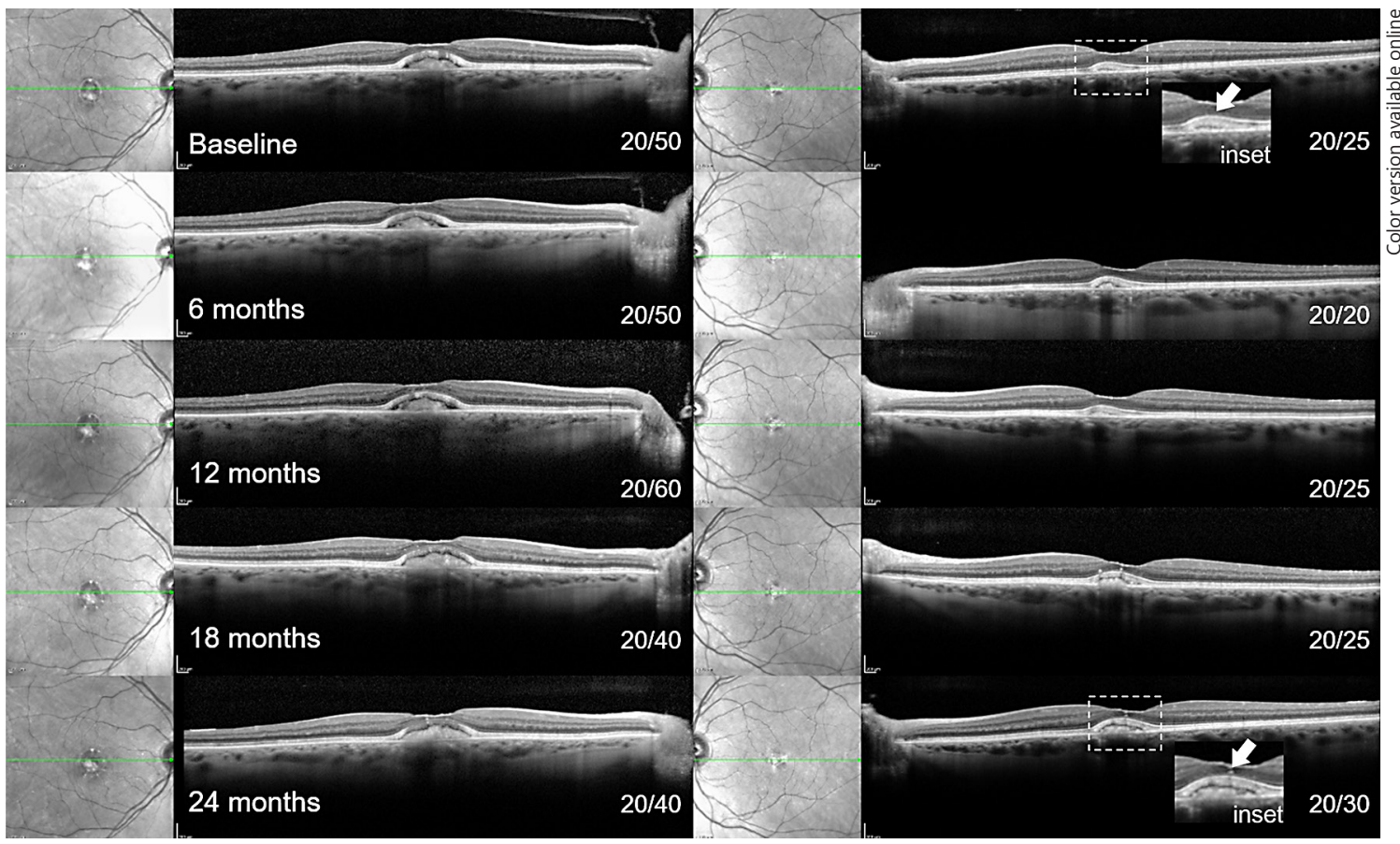

Fig. 4. Serial spectral-domain optical coherence tomography imaging from baseline (pre-sildenafil) to 24 months in 6-month intervals in patient 3. Acquired vitelliform lesions are apparent in the subretinal space of both eyes underlying an intact or intermittently intact ellipsoid zone layer, which remained stable along with visual acuity over 2 years (inset).

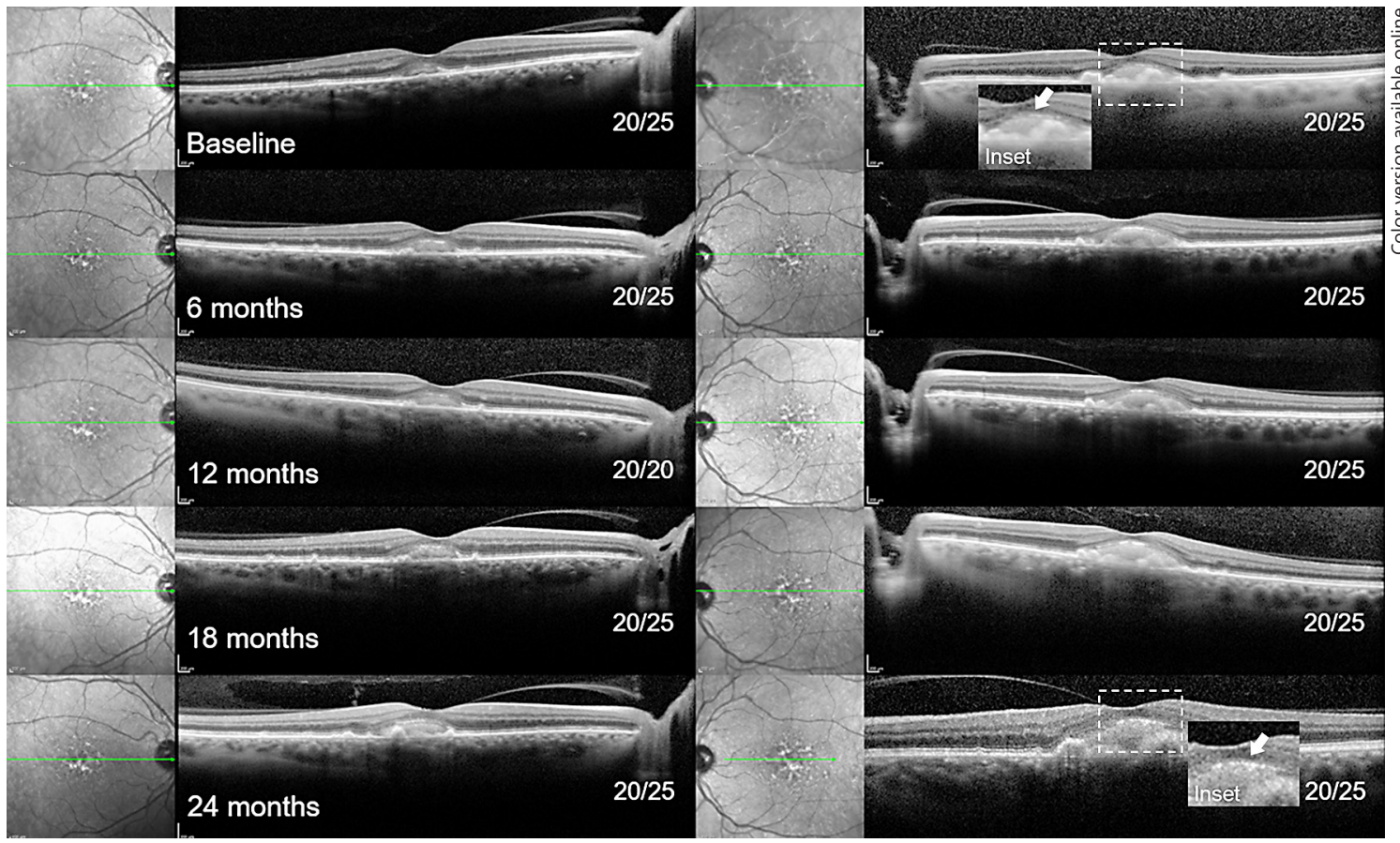

Fig. 5. Serial spectral-domain optical coherence tomography imaging from baseline (pre-sildenafil) to 24 months in 6-month intervals in patient 4. An acquired vitelliform lesion is apparent in the subretinal space of both eyes. The ellipsoid zone layer (inset, white arrow) became increasingly discernable over time, with no significant changes in visual acuity.

Sildenafil Treatment for Macular Degeneration
Ophthalmologica

DOI: $10.1159 / 000486105$ 


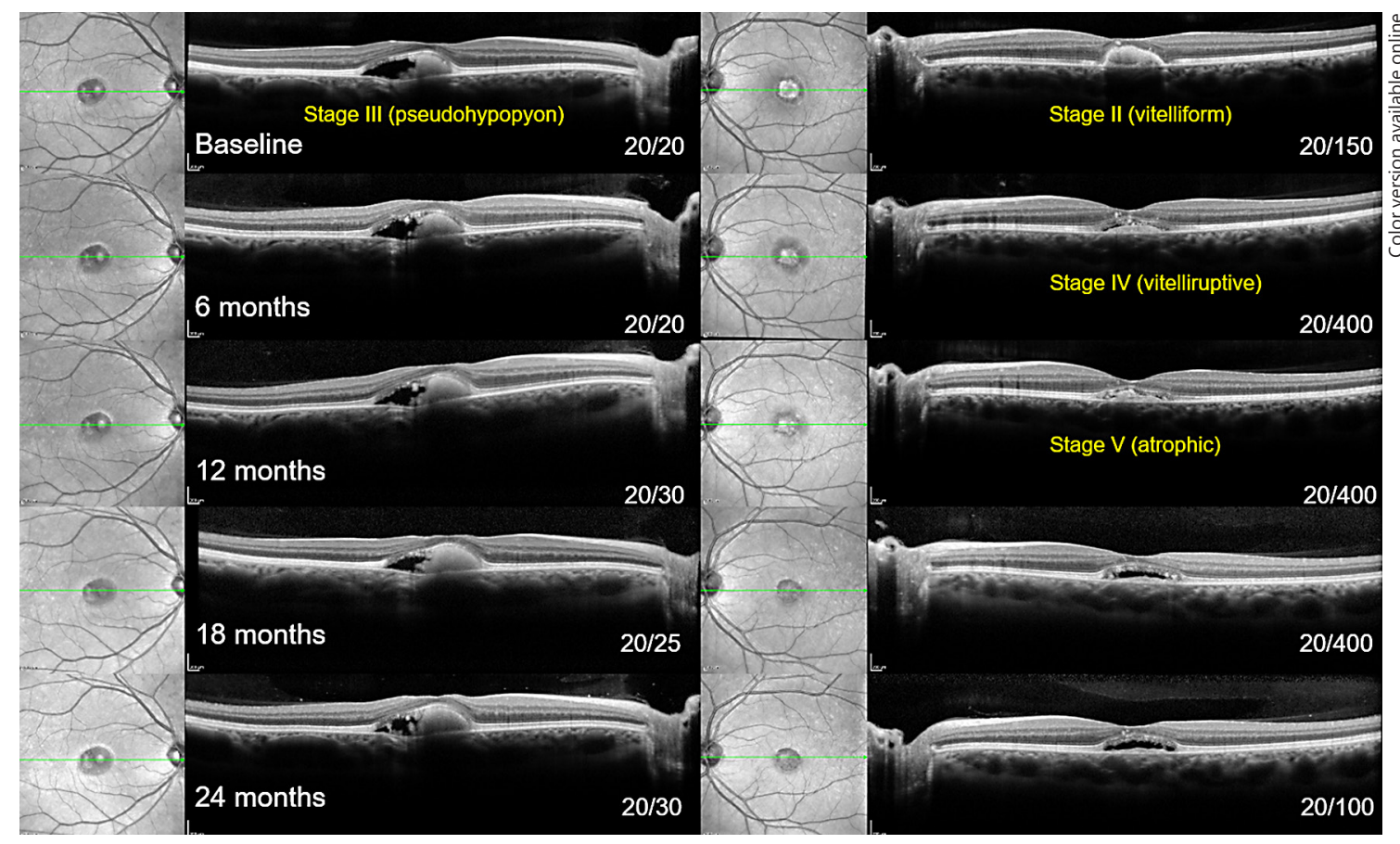

Fig. 6. Serial spectral-domain optical coherence tomography imaging from baseline (pre-sildenafil) to 24 months in 6-month intervals in Best disease (patient 5). The right eye exhibited a pseudohypopyon (stage III) vitelliform lesion at presentation which remained stable over time, while the left eye initially presented at stage II and progressed to the atrophic stage after 1 year with an accompanying decline in visual acuity from 20/150 to 20/400. Visual acuity subsequently recovered to $20 / 100$ by the second year of follow-up.

All participants self-reported improvements in contrast sensitivity, and though we saw no significant change in the Pelli-Robson chart analysis, the Best disease patient could now see the chart with both eyes.

\section{Discussion}

Sildenafil inhibits PDE5 and PDE6 in the choroid, and this inhibition increases choroidal perfusion [7]. PDE6 inhibition decreases Warburg glycolysis (Fig. 9) in rod photoreceptors, which in turn promotes lipid biosynthesis through parasympathetic mechanisms [15]. Excessive Warburg glycolysis in photoreceptor leads to an increase in retinal pigment epithelium stress and thickening of Bruch's membrane [16]. Hypoxia-induced metabolic stress in retinal pigment epithelium cells is sufficient to induce photoreceptor degeneration [17].

The results suggest a beneficial effect, but do not prove that the choroidal vessel-filling materially effects the choriocapillaris, which in some OCT angiography studies appeared to remain full even in dry AMD [18]. Therefore, the potential of a barrier, such as a thickened Bruch's membrane, may limit the potential value of the choroidal flow. Bird [19] referred to thickening of Bruch's membrane as a hindrance to proper metabolic transfer and ischemia-induced degeneration. Clearly however, the PDE6 effect remains significant, both for decreasing Warburg glycolysis and perhaps inhibiting thickening of Bruch's membrane as well as promoting recovery of the ellipsoid layer.

Future studies to clarify this barrier are required and may ultimately indicate earlier treatment with PDE6 inhibitors. Perhaps patients with early detectable drusen should be considered for sildenafil therapy, particularly if there is a significant family history of degeneration. Since PDE6 effectiveness is related to the light phase of Warburg glycolysis, it may be helpful to prescribe sildenafil in daytime rather than twice a day since the day phase increases the PDE5 effected by approximately 100 times [20].

The effects of perfusion in the younger, softer drusen (those associated with AMD) seem obvious, though less so in the older calcified drusen. The ultrasound measurement does not significantly relate to different choroidal 

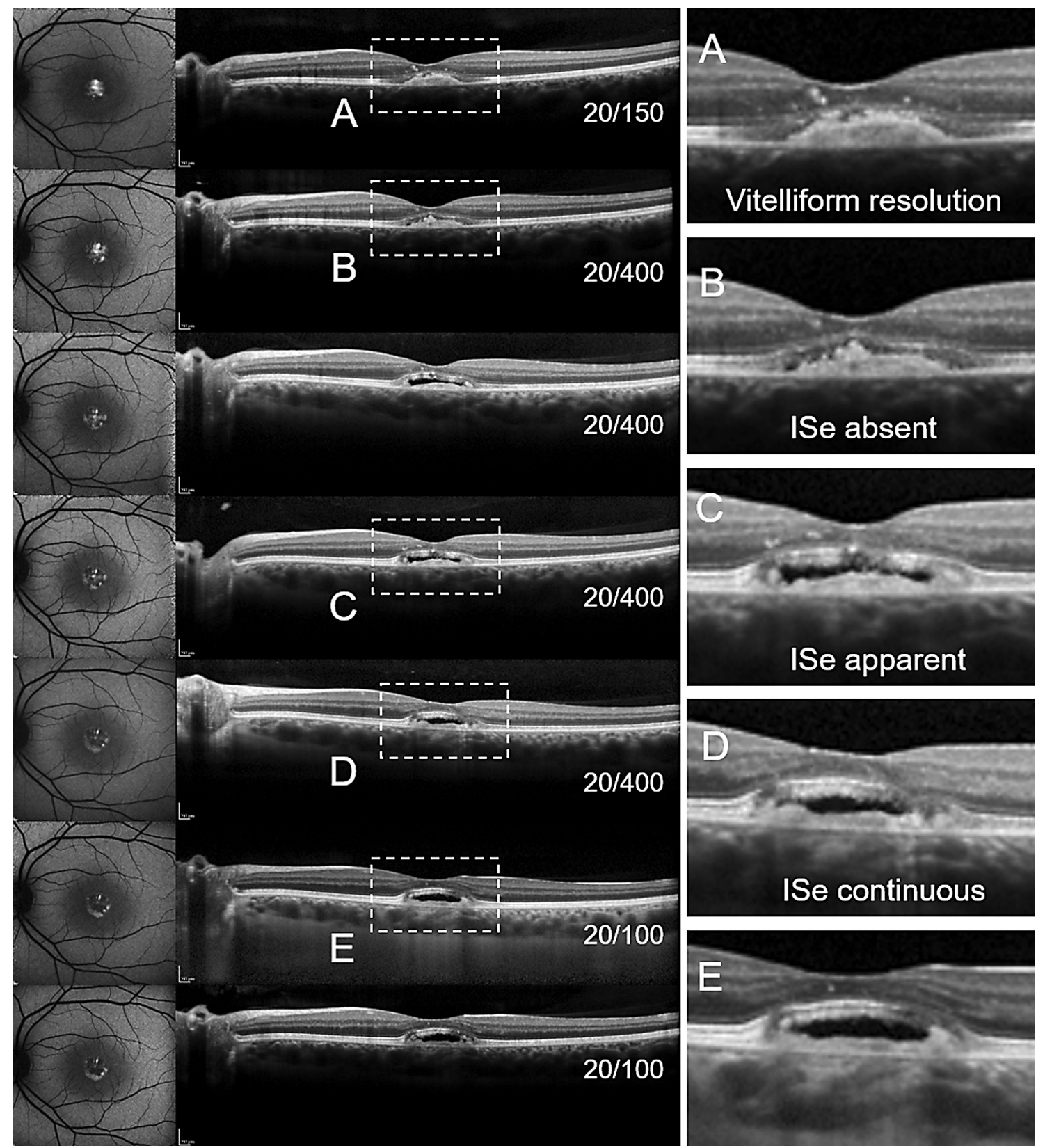

Fig. 7. Visual acuity progression and corresponding spectral-domain optical coherence tomography scans illustrating the loss and subsequent visibility of the ellipsoid zone band in patient 5.

layers, only perfusion within those layers. As a result, the values can only be suggested as adjunctive in dry AMD; however, they appear to be a positive therapy in newer softer lesions such as are present in the soft stages of vitelliruptive macular degeneration and Best vitelliruptive macular degeneration. In a case study involving 5 years of sildenafil treatment in a patient with late-onset vitelliruptive macular degeneration (submitted for publication), we observed longitudinal safety of sildenafil, arrest of vision deterioration, and sustained improvements of BCVA.

It is notable that these participants remained visually stable, given that in macular degeneration there would be an expected loss of vision over the course of 24 months, and that a significant improvement in vision was measured by BCVA in the participant with Best vitelliruptive macular degeneration. 


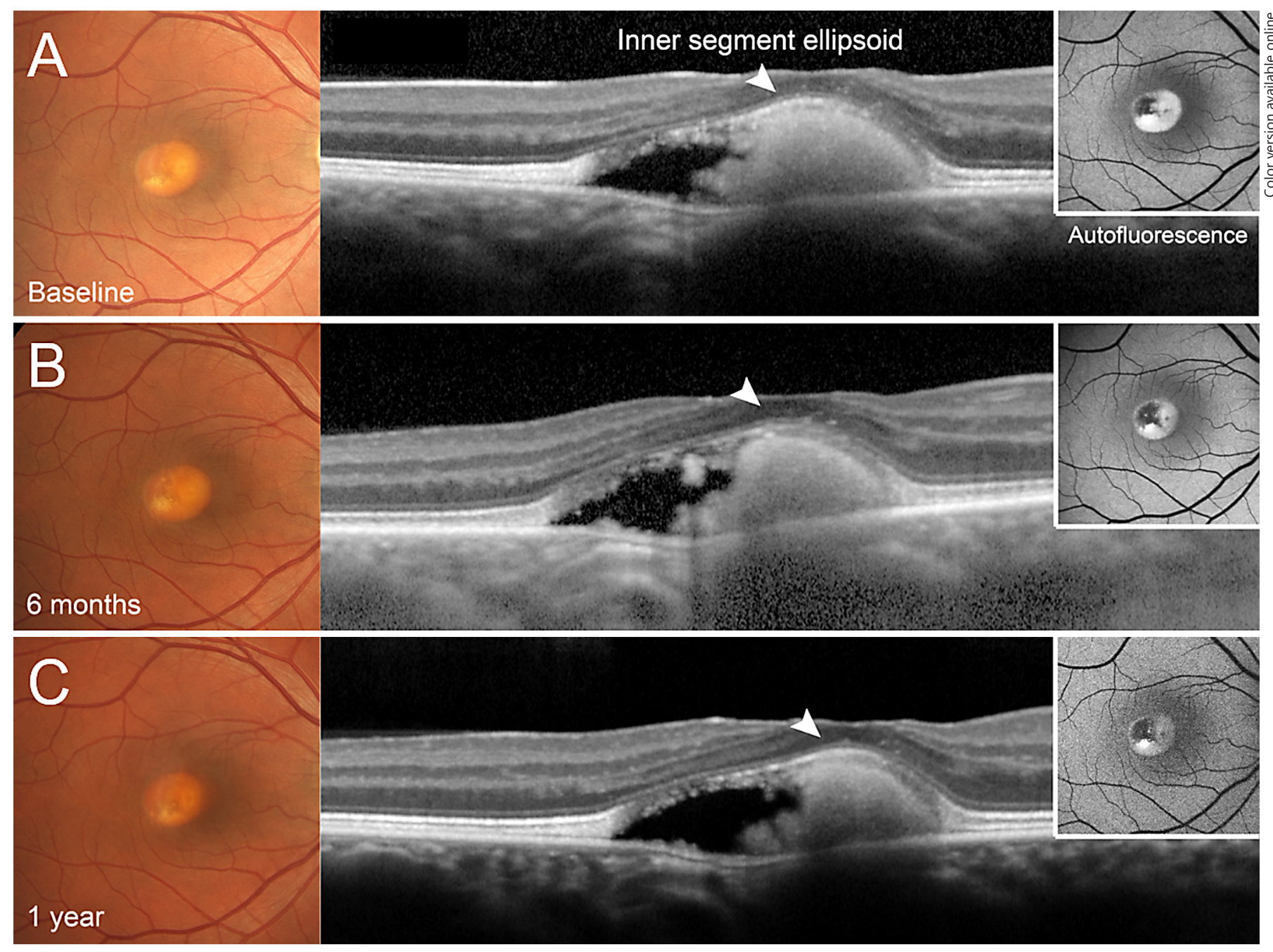

Fig. 8. Color fundus photographs and corresponding spectral-domain optical coherence tomography and autofluorescence imaging in Best disease (patient 5) illustrating the stable presence of the ellipsoid zone band anteriorly situated over the lesion.

Accurate measurement of perfusion in the choroid is challenging as it would entail measurement of both the luminal diameters of the choroidal vessels and their blood flow velocities. We have shown that sildenafil mediates choroidal flow by determining the fraction of the choroid occupied by measurable flow (decorrelation) using the swept-mode ultrasound imaging technique as a proxy for choroidal perfusion [21]. A similar processing scheme is currently used in split-spectrum OCT angiography to define the vascular density (the percentage area occupied by blood vessels) having at least two standard deviations above noise [22]. Most recently, ultrafast ultrasound imaging has been demonstrated to provide depictions of choroidal flow dynamics, including velocities over the cardiac cycle [21]. These imaging techniques provide documented substantiation of increased choroidal perfusion [22]. Overall, these techniques show growing promise for even more precise measurements of choroidal perfusion in the future and should be developed further.

While these representative cases are of a small sample size, they are consistent with two prime observations. First, vision is largely maintained even though no direct evidence of maintenance of perfusion in the choriocapillaris is possible and no significant reduction in drusen is observed. Second, and more importantly, the improvement and recovery of the photoreceptor layer is consistent with PDE6 inhibition. 


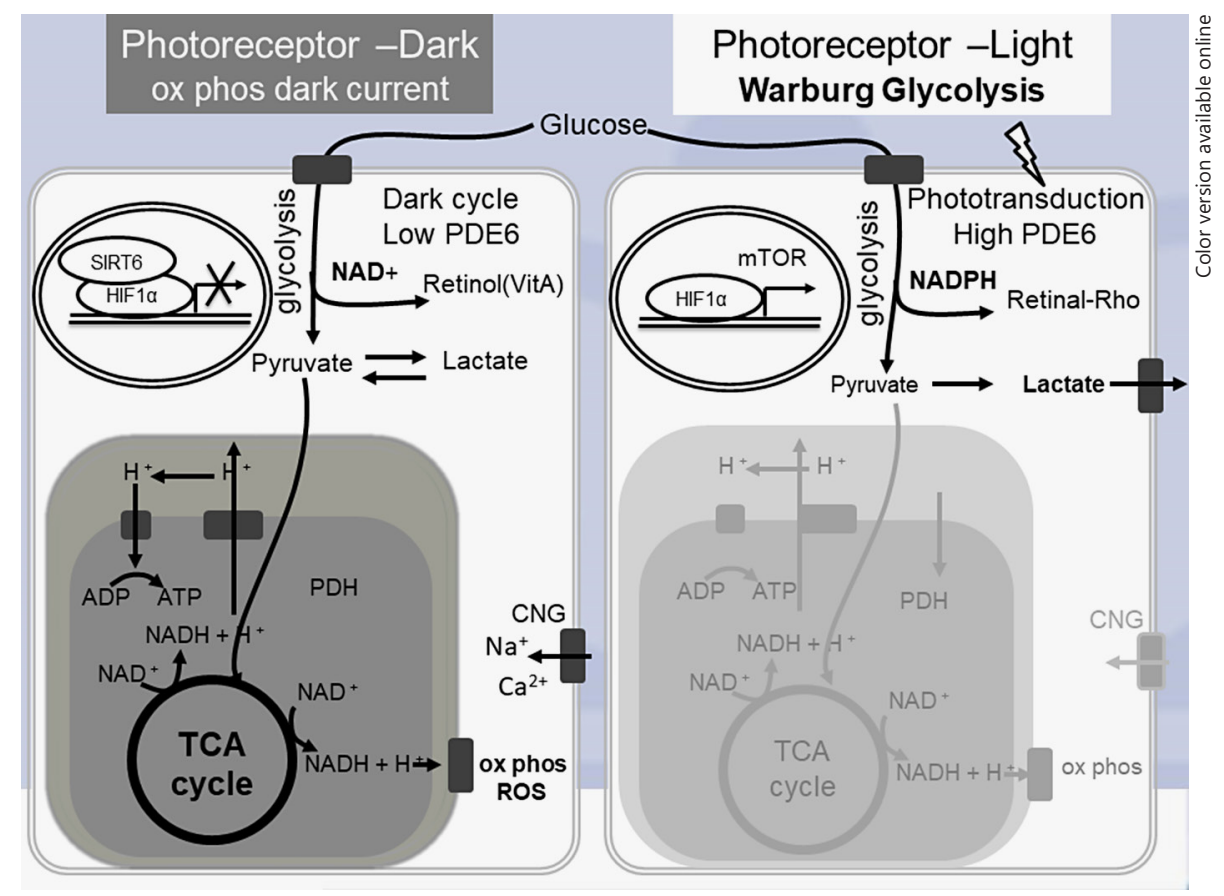

Fig. 9. Photoreceptor metabolism cycles between an anaerobic light cycle and an aerobic dark cycle associated with phototransduction through PDE6 activities and mediated by SIRT6 and HIF1a. In the dark cycle, photoreceptor metabolism is similar to neurons. Glucose is metabolized via oxidative phosphorylation, generating NADH/NADPH, ATP to fuel the CNG channels for continuous depolarization to activate secondary neurons, and also producing ROS. SIRT6 acts as the driver of aerobic respiration; SIRT6 inhibits HIF1a to prevent transcription of glycolysis enzymes including PDK and PFK-1 and to promote oxidative phosphorylation. In the light cycle, photoreceptor metabolism is similar to that of cancer cells in the Warburg effect. Glucose undergoes glycolysis to form pyruvate and lactic acid, and glucose also enters

As proposed here and by others $[23,24]$, the parasympathetic control of the choroid, in particular the choriocapillaris, might further illuminate the impact of sildenafil on the choroid tissue and be considered a future direction for research. Additionally, because of the solid safety record of sildenafil thus far, consideration might be given to larger doses of sildenafil to improve results. Increased perfusion may also be helpful in other lesions or treatments such as stem cell therapies, where an enhanced vascular flow could be clinically beneficial.

These preliminary data support the use of sildenafil and a further assessment in a larger study. Further refinement of the evaluation of these effects would be enhanced by including microperimetry and OCT angiography in longitudinal studies particularly as we search for other systemic treatments.

Sildenafil Treatment for Macular

Degeneration the pentose phosphate pathway, generating several molecules of NADPH. NADPH is used for anabolic production of fatty acids, proteins, nucleic acids, and 11-cis retinal. Light activates the phototransduction pathway through high PDE6, and energy consumption is 4 times less than in the dark cycle. ADP, adenosine diphosphate; ATP, adenosine triphosphate; HIF1a, hypoxia-inducible factor $1 \alpha$; ROS, reactive oxygen species; TCA, tricarboxylic acid; NADH, nicotinamide adenine dinucleotide (reduced); $\mathrm{NAD}^{+}$, nicotinamide adenine dinucleotide (oxidized); PDK, pyruvate dehydrogenase kinase; PFK-1, phosphofructokinase-1; NADPH, nicotinamide adenine dinucleotide phosphate; Ser, serine; Gly, glycine; GSSG, glutathione disulfide; GSH, glutathione.

\section{Acknowledgment}

Jonas Children's Vision Care and Bernard and Shirlee Brown Glaucoma Laboratory are supported by grants from the National Institutes of Health (5P30EY019007, R01EY018213, R01EY024698, R01EY026682, R21AG050437), National Cancer Institute Core (5P30CA013696), the Research to Prevent Blindness (RPB) Physician-Scientist Award (S.H.T.), unrestricted funds from RPB, New York, NY, USA, and Triad Foundation, Ithaca, NY, USA. Writing services were provided by Jim Sliney Jr., RMA (Columbia University, New York, NY, USA).

\section{Disclosure Statement}

No conflicting relationship exists for any of the authors. 


\section{References}

1 Seddon JM, Reynolds R, Yu Y, Daly M J, Rosner B: Risk models for progression to advanced age-related macular degeneration using demographic, environmental, genetic, and ocular factors. Ophthalmology 2011;118: 2203-2211.

-2 Chew EY, Clemons TE, Agrón E, Sperduto RD, Sangiovanni JP, Davis MD, Ferris FL 3rd; Age-Related Eye Disease Study Research Group: Ten-year follow-up of age-related macular degeneration in the Age-Related Eye Disease Study: AREDS report No. 36. JAMA Ophthalmol 2014;132:272-277.

$>3$ Hayreh SS: Ischemic optic neuropathy. Prog Retin Eye Res 2009;28:34-62.

4 Friedman E, Oak SM: Choroidal microcirculation in vivo. Bibl Anat 1965;7:129-132.

5 Flower RW, von Kerczek C, Zhu L, Ernest A, Eggleton C, Topoleski LD: Theoretical investigation of the role of choriocapillaris blood flow in treatment of subfoveal choroidal neovascularization associated with age-related macular degeneration. Am J Ophthalmol 2001;132:85-93.

6 Grunwald JE, Siu KK, Jacob SS, Dupont J: Effect of sildenafil citrate (Viagra) on the ocular circulation. Am J Ophthalmol 2001;131:751755.

$>7$ Coleman DJ, Silverman RH, Rondeau MJ, Lloyd HO, Khanifar AA, Chan RVP: Age-related macular degeneration: choroidal ischaemia? Br J Ophthalmol 2013;97:1020-1023.

$>8$ Kim DY, Silverman RH, Chan RV, Khanifar AA, Rondeau M, Lloyd H, Schlegel P, Coleman DJ: Measurement of choroidal perfusion and thickness following systemic sildenafil (Viagra ${ }^{\circledR}$ ). Acta Ophthalmol 2013;91:183188.
-9 Vance SK, Imamura Y, Freund KB: The effects of sildenafil citrate on choroidal thickness as determined by enhanced depth imaging optical coherence tomography. Retina 2011;31:332-335.

10 Harris A, Kagemann L, Ehrlich R, Ehrlich Y, Lopez CR, Purvin VA: The effect of sildenafil on ocular blood flow. Br J Ophthalmol 2008; 92:469-473.

11 Kurtulan E, Gulcu A, Secil M, Celebi I, Aslan G, Esen AA: Effects of sildenafil on ocular perfusion demonstrated by color Doppler ultrasonography. Int J Impot Res 2004;16:244248.

12 Galiè N, Ghofrani HA, Torbicki A, Barst RJ, Rubin LJ, Badesch D, Fleming T, Parpia T, Burgess G, Branzi A, Grimminger F, Kurzyna M, Simonneau G; Sildenafil Use in Pulmonary Arterial Hypertension (SUPER) Study Group: Sildenafil citrate therapy for pulmonary arterial hypertension. N Engl J Med 2005;353:2148-2157.

13 Wirostko BM, Tressler C, Hwang LJ, Burgess G, Laties AM: Ocular safety of sildenafil citrate when administered chronically for pulmonary arterial hypertension: results from phase III, randomised, double masked, placebo controlled trial and open label extension. BMJ 2012;344:e554.

14 Cordell WH, Maturi RK, Costigan TM, Marmor MF, Weleber RG, Coupland SG, Danis RP, McGettigan JW Jr, Antoszyk AN, Klise S, Sides GD; ERG Testing During Chronic PDE5 Inhibitor Administration (ERGPDE5i) Consortium: Retinal effects of 6 months of daily use of tadalafil or sildenafil. Arch Ophthalmol 2009;127:367-373.

$>15$ Steinle JJ, Krizsan-Agbas D, Smith PG: Regional regulation of choroidal blood flow by autonomic innervation in the rat. Am J Physiol 2000;279:R202-R209.
16 Lin MK, Kim SH, Zhang L, Tsai YT, Tsang $\mathrm{SH}$ : Rod metabolic demand drives progression in retinopathies. Taiwan J Ophthalmol 2015;5:105-108.

-17 Kurihara T, Westenskow PD, Gantner ML, Usui Y, Schultz A, Bravo S, Aguilar E, Wittgrove C, Friedlander MS, Paris LP, Chew E, Siuzdak G, Friedlander M: Hypoxia-induced metabolic stress in retinal pigment epithelial cells is sufficient to induce photoreceptor degeneration. Elife 2016;5:e14319.

18 Chen JC, Fitzke FW, Pauleikhoff D, Bird AC: Functional loss in age-related Bruch's membrane change with choroidal perfusion defect. Invest Ophthalmol Vis Sci 1992;33:334-340.

19 Bird AC: Doyne Lecture. Pathogenesis of retinal pigment epithelial detachment in the elderly; the relevance of Bruch's membrane change. Eye (Lond) 1991;5:1-12.

20 Okawa H, Sampath AP, Laughlin SB, Fain GL: ATP consumption by mammalian rod photoreceptors in darkness and in light. Curr Biol 2008;18:1917-1921.

21 Urs R, Ketterling JA, Silverman RH: Ultrafast ultrasound imaging of ocular anatomy and blood flow. Invest Ophthalmol Vis Sci 2016; 57:3810-3816.

22 Chalam KV, Sambhav K: Optical coherence tomography angiography in retinal diseases. J Ophthalmic Vis Res 2016;11:84-92.

23 Marmor MF, Kessler R: Sildenafil (Viagra) and ophthalmology. Surv Ophthalmol 1999; 44:153-162.

24 Laties AM: Vision disorders and phosphodiesterase type 5 inhibitors: a review of the evidence to date. Drug Saf 2009;32:1-18. 